\title{
The prevalence of selected buoyancy alternate conceptions at two colleges
}

\author{
DJ Wagner ${ }^{1}$ and Peter S. Shaffer ${ }^{2}$ \\ ${ }^{1}$ Department of Physics, Grove City College, 100 Campus Drive, Grove City, PA, 16127 \\ ${ }^{2}$ Department of Physics, University of Washington, Box 351560, Seattle, WA, 98195
}

\begin{abstract}
Review of prior studies, mostly at the K-12 level, along with long-answer responses and interviews given to undergraduates at Grove City College, have identified over 150 alternate conceptions about buoyancy. In order to probe how prevalent some of these are in the college-age population, we designed and administered multiple-choice and free-response questions to students at both the University of Washington and Grove City College. This paper presents some of those results.
\end{abstract}

\section{INTRODUCTION}

Studies dating back at least to Piaget [1] have examined how people think about buoyancy. A review of published research, and our own investigations, have identified a plethora of alternate conceptions [2], most of which have been studied only in K-12 populations. The few studies involving more advanced students have tended to focus primarily on density and buoyant force [3]. We have been examining the prevalence in college-aged populations of the numerous buoyancy-related conceptions, in part because one of the authors is developing a standardized assessment for buoyancy and deciding which distractors to include.

Several alternate conceptions identified in our investigation, such as "sticky liquids make things float" [4], seemed unlikely to be held by a large fraction of college students. Yet our investigations revealed that even these seemingly naïve conceptions were given by a non-negligible fraction of college students. This paper reports on the prevalence of four of these expectation-confounding conceptions in several populations at two institutions, demonstrating the risk of dismissing the significance of an alternate conception based on one's initial judgment.

\section{METHODOLOGY}

Questions probing the selected conceptions were developed and asked at both the University of Washington (UW), a large public research-one university, and Grove City College (GCC), a small private 4-year liberal arts college. Differing institutional norms required the assessment protocol to be different at each institution.

At UW, versions of the questions were included as part of online "pretests" administered over multiple quarters of the calculus-based sequence (UW Calc). These pretests were given before students had worked through a tutorial [5] on pressure. The lectures had covered pressure, but not buoyancy, so the assessment was during instruction on fluids. The questions were in multiple-select format, often followed by a request for explanations of reasoning.

At GCC, the questions were included on two versions of a multiple-select bubble-sheet assessment given at the beginning and end of the semester in calculus-based (GCC Calc), trigonometry-based (GCC Trig), and conceptual courses (GCC Cncp). To keep the total assessment length reasonable, explanations were requested for only a few selected questions. Instruction in each course included a 2hour workshop either using or inspired by a tutorial [5] on buoyancy.

\section{CONCEPTIONS, QUESTIONS, AND DATA}

Each of the four targeted conceptions is described in detail below, along with the questions used to probe their prevalence, and the results from those questions. Most of these questions also probe other ideas not specific to this paper; those results are not discussed. Students who did not respond to a given question were omitted from the analysis of that question. Thus the population sizes reported in the tables vary slightly by question.

The tables showing the results use the abbreviations W16, S16, Su16, and F16 for Winter, Spring, Summer, and Fall 2016, respectively. Pre, Post, and Mid denote when during instruction each assessment was given. Different versions administered during the same term are shown as vA and vB. Administering multiple versions of the assessment allowed us to maximize the number of conceptions that could be probed in the available time but resulted in unmatched pre/post data. We therefore can only claim that certain conceptions exist after instruction and do not make any claims about the effects of instruction.

\section{A. "Sticky liquid"}

The first conception is identified as "Sticky liquids make things float" in a study of middle-school students [4]. That study reports no other description, and we wanted both to find the prevalence of this idea among college students and to investigate the reasoning associated with this idea. We asked a set of three questions based on a closed jar containing just enough marbles to remain fixed when released underwater, as illustrated in Fig. 1.

Students were told to treat liquids as incompressible and that cooking oil is less dense and 'stickier' than water. The questions used included (1) What would be the final location of the object if the water were replaced by oil?; (2) Which of a set of justifications (Fig. 1) did you use in answering the previous question?; and (3) How did each justification lead to your conclusion about the final location of the jar in oil?

Figure 2 shows the fraction of students including option A (the "sticky" option) in their choices for the Jar question. 


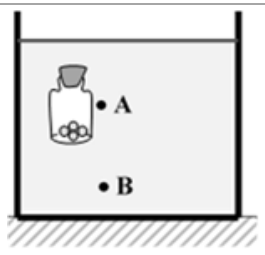

When deciding the location of the jar when the water is replaced by oil, which of the following possible justifications did you use?

Select all that apply.

A. Vegetable oil is "stickier" than water.

B. Water has a higher density than vegetable oil.

C. Floating objects always float.

D. The weighted jar must be denser than water.

E. Objects will float at equilibrium at a specific depth determined by the mass of the object.

F. Other (please detail in explanation).

FIG 1. Image accompanying, and justification for, the Jar question.

For most of the populations, this fraction is near or above $10 \%$, exceeding $25 \%$ for students before instruction in the conceptual course at GCC.

The long-answer portion of the Jar question was included to provide insight into how students reason about the effect of "stickiness" on sinking and floating. Although most explanations failed to mention stickiness or simply restated that it mattered, a few responses gave additional insight. Moreover, even some students who hadn't chosen the "sticky" option mentioned stickiness in their explanations.

Some students argued that the stickiness provided a buoyant effect, yet others argued for an "anti-buoyant" (enhanced sinking) effect. Explanations claiming stickiness assists floatation include, "Since vegetable oil is 'stickier' than water, it would cling [to the jar], preventing it from sinking..." Another student took this to the extreme: "[the jar remains fixed at A in oil] because cooking oil is sticky."

One student who only chose "Other" (choice F) as a justification for the jar sinking in oil explained the antibuoyant sticky effect as follows: "... oil is more adhesive than cohesive, so it will be easier for objects to move through

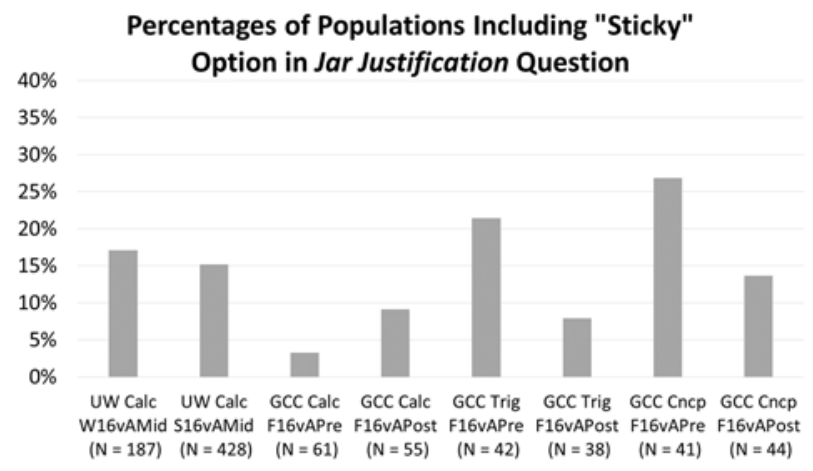

FIG 2. Prevalence of targeted conception for each population on the Jar question.
A student half fills a container with marbles and screws on the lid. The container with marbles sinks when placed in an aquarium filled with water. Which of the following might result in the container floating? Select all that apply.

A. Filling the other half of the container with cork.

B. Removing the marbles.

C. Placing a helium-filled balloon in the other half of the container.

D. Making the container "flatter" by tipping the container on its side.

E. Surrounding the container with more water, by using a wider aquarium, filled just as deep.

FIG 3. The Marbles question.

oil molecules than it will be for objects to move through water molecules."

In a prior study by one of the authors [2], an interview subject expressed an animistic quality to anti-buoyant stickiness: "I thought of the molasses swamp in Candy Land ... perhaps because [maple syrup is] sticker it might be just like [suction sound effect] 'oh you're mine."'

\section{B. "Inherent floatability"}

Another conception found in the literature (see, for example, Ref. [4]) is that floatability is an inherent property of a substance. For example, some students seem to believe that a "floater," like cork, placed inside a sunken object will cause the object to move to the surface. The Marbles question (Fig. 3) was used to examine the prevalence of this idea.

As shown in Fig. 4, more than $15 \%$ of the students in each population thought adding cork inside the container (option A) could cause the container to float. Explanations expressed the idea that cork has "inherent" buoyancy: "cork has a greater buoyancy so it would increase the buoyancy of the system." This conception seems almost pre-causal: cork is animistic in its ability to lift the object. Some explanations also indicated difficulty with the idea of density. Several students said that adding cork decreases the density of the

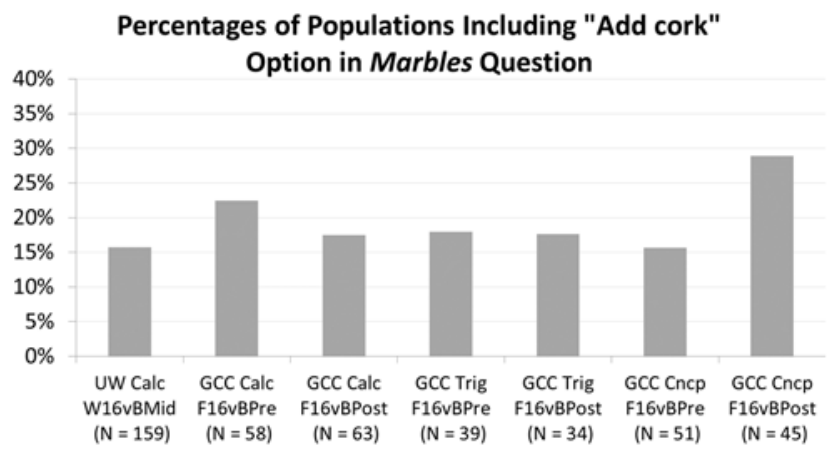

FIG 4. Prevalence of targeted conception for each population on the Marbles question. 
Consider Object B, which floated. Imagine cutting Object B horizontally along the water line, separating part " 1 " under the water line from part " 2 " above, as shown. Which of the following is a correct statement? Select all that apply.

A. The density of part 2 will be greater than the density of part 1.

B. The density of part 1 will be greater than the density of part 2 .

C. Part 2 might sink to the bottom.

D. Part 1 might sink to the bottom.

E. The mass of part 1 will be greater than the mass of part 2 .

F. The mass of part 2 will be greater than the mass of part 1 .

G. None of the above.

\section{FIG 5. The Cut object question.}

object, e.g., "cork might counteract the weight of the marbles," and "filling the container with cork ... can make the density of the object decrease ...."

\section{C. "Water line divides floating/sinking parts of floaters"}

A conception identified in a study of 7-14 yr old students [6] is related to the behavior of an object that floats partially submerged. Nearly half of the 76 interview subjects claimed that cutting off the part of the object above the water would result in the bottom portion sinking.

To determine whether this confusion persisted among college-level students, we asked the Cut object question shown in Fig. 5. The question was prefaced by a statement that object $B$ is solid and made of a single material. Note that this question probes several different student ideas about floating and density; however, for the purpose of this paper, options C and D are the most relevant. Students who believe that the top part of the object floats and the bottom part sinks should choose option D (the bottom part might sink) and not choose option $\mathrm{C}$ (the top part might sink).

The graph in Fig. 6 shows the fraction of noncontradictory, non-null responses that included option D (the bottom might sink) but not option $\mathrm{C}$ (the top might sink). (A contradictory response was considered to be one that included incompatible choices, i.e., both $\mathrm{A}$ and $\mathrm{B}$ or both $\mathrm{E}$

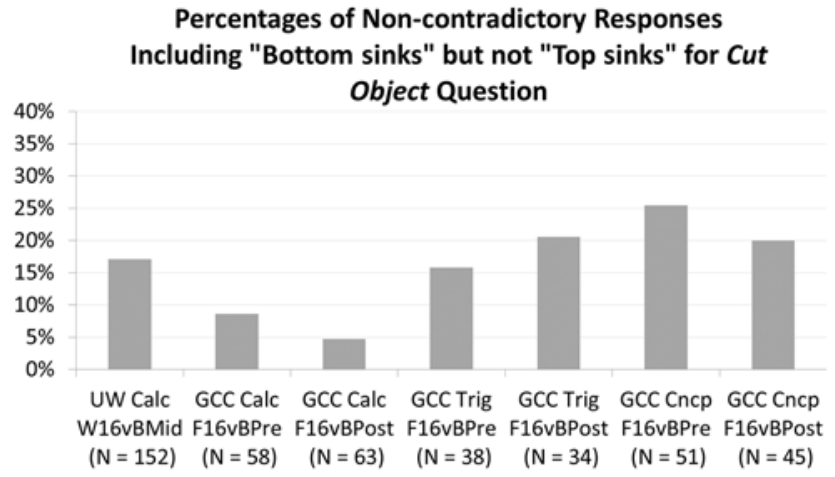

FIG 6. Prevalence of targeted conception for each population on the Cut object question. and F.) More than $15 \%$ of all populations except the GCC calc-based class gave such a sink/float response.

\section{D. "Direction of buoyant force depends on depth"}

The final targeted conception is the belief that the direction of the net force by water changes with depth. An interview subject in a prior study conducted at GCC [2] knew "from personal experience" that the force exerted by water on a person is upward when that person is just below the surface but changes to downward as the person moves deeper.

We probed this conception through the Triangles question, which involves three submerged triangles suspended by strings. (See Fig. 7.) Triangles B and C have the same orientation but are at different depths. Students were asked to indicate the direction of the "total force exerted by the water on each object" and to choose the justification(s) for their answer from the list in Fig. 7.

The options for the justification question in Fig. 7 were modified between the UW, GCC pre-instruction, and GCC post-instruction offerings. The UW version, which was given first, had fewer choices, but based on the explanations given by some students, we added two choices (A and $\mathrm{D}$ ) for the GCC pre-instruction version. Based on student explanations in that offering we added option $G$ and modified the text for option $\mathrm{B}$ for the post-instruction offering. (Option B in the $\mathrm{UW}$ and $\mathrm{GCC}$ pre-instruction
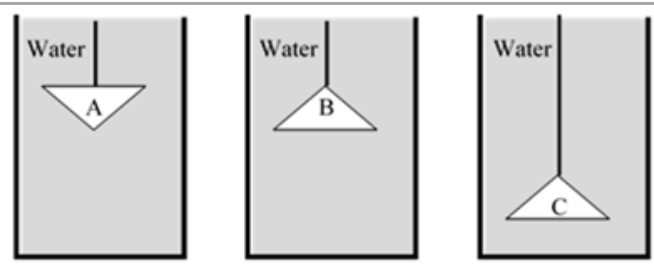

Which of the following possible justifications did you use to decide whether the forces exerted by water on objects $\mathbf{B}$ and $\mathbf{C}$ are in the same direction, opposite directions, or zero? Select all that apply.

A. The objects are not accelerating, so the water does not exert a force on the object.

B. The direction of the force exerted by the water is affected by the object's depth under the surface, or the amount of water above and below the object.

C. The direction of the force exerted by the water is affected by the base area of the object.

D. Both objects are moving downward, so the direction of the forces on them is the same.

E. The direction of the force exerted by the water is affected by the direction the object sides are slanted.

F. The direction of the force exerted by the water is affected by the tension in the string supporting the object.

G. The force exerted by the water is a reaction to other forces acting on the object, so its direction depends upon the direction of other forces.

$\mathrm{H}$. The direction of the force exerted by the water is independent of object shape, orientation, tension forces, and depth.

FIG 7. Image accompanying, and justifications for, the Triangles question. 


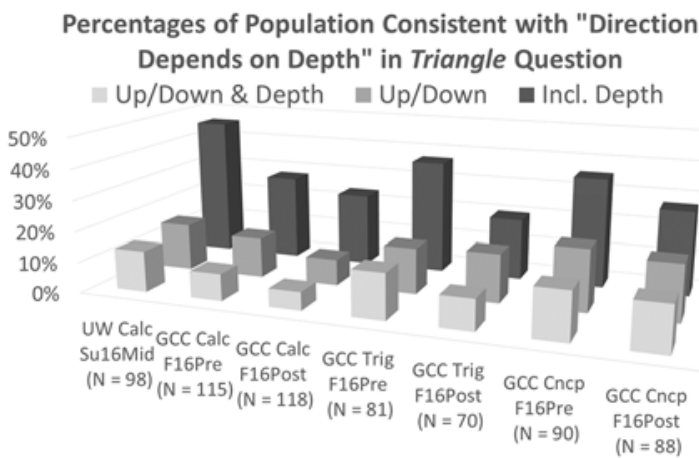

FIG 8. Prevalence of different measures of the targeted conception for each population on the Triangles question.

versions read, "The direction of the force exerted by the water is affected by the object's depth under the surface.") The differences in the questions may have affected student responses to some extent, but in this study we are only trying to determine whether the four alternate conceptions are prevalent among college-age students. We are not comparing results from different populations or at different stages of instruction. Thus the changes do not affect our conclusions.

We examined two ways that students could indicate a belief that the force by the water changes direction, from up to down, as the depth of the object increases. On the first part of the question (Fig. 7) students could have chosen an upward force for triangle $\mathrm{B}$ and a downward force for triangle C. They could also have included option B (the direction depends on depth) in their response to the justification question. Some students did only one or the other, some students did both.

Figure 8 shows three different measures of the targeted conception. The back, largest, set of bars (labeled 'Incl. Depth') shows the percentage of each population that included option B (the direction of the force by the water depends on depth) in their response to the justification question (Fig. 7). Between about $20 \%$ and $45 \%$ of each population gave this answer. The middle bars ('Up/Down,'), which range from about $10 \%$ to $20 \%$, represent students who said the force is upward for triangle B and downward for triangle $\mathrm{C}$ (Fig. 7). The front set of bars (ranging from $5 \%$ to $15 \%$ ) show students who were consistent on the two questions, indicating both that the forces on triangles B and
$\mathrm{C}$ are up and down, respectively, and who included option B in their justification.

\section{CONCLUSIONS}

The first three conceptions examined in this study ("Sticky liquid," "Inherent floatability," and "Water line divides floating/sinking parts of floater") had been documented among precollege students. The fourth ("Direction of water force depends on depth") had been seen in only one interview with a university-level student, but that student had been strongly convinced of his reasoning. At the onset of this study, we had not anticipated that any of these conceptions would be present at a significant level among university students, in particular after instruction. However, in preparing a standardized instrument to assess student understanding of buoyancy, we regarded it as important to know whether these ideas could reasonably be excluded for consideration when choosing the options used as distractors.

Contrary to our expectations, each of these alternative conceptions was present among a non-negligible fraction (at or above the $10 \%$ level) of most of our populations. In particular, the idea of "Inherent floatability" was uniformly present, seen in at least $15 \%$ of all populations. The results of the Triangles question illustrate that student responses can often be inconsistent and context-dependent, so a single question may not necessarily provide a robust measurement of the students holding a particular conception.

The results of this investigation can serve as a reminder, even for experienced instructors and physics education researchers, that university students (including those in calculus-based courses) may hold what can seem like relatively naïve ideas. Research is critical for assessing the beginning and ending states of student understanding and for developing curriculum that meets the needs of all students in our courses.

\section{ACKNOWLEDGEMENTS}

The authors thank the following student members of the GCC and UW PEG groups who contributed to this project: Shannon Armstrong, Sheh Lit Chang, Kristen Hephner, Randon Hoselton, and Mercedes Mansfield. Partial support has come from the GCC Swezey Scientific Instrumentation and Research Fund and NSF DUE 1022449.
[1] J. Piaget, The Child's Conception of Physical Causality, translated by M. Gabain (Kegan Paul \& Co., London, 1930).

[2] D. Wagner, poster PST1D35 presented at 2016 Summer meeting of American Association of Physics Teachers; paper in preparation.

[3] M. E. Loverude, C. H. Kautz, and P. R. L. Heron, Am. J. Phys. 71, (11), 1178-1187 (2003); D. Wagner, E. Carbone, and A. Lindow, in 2013 Physics Education
Research Conference, edited by P. V. Engelhardt, A. D. Churukian, and D. L. Jones (Portland, OR, 2013).

[4] Y. Yin, M. K. Tomita, and R. J. Shavelson, Sci. Scope 31 (8), 34-39 (2008).

[5] L. C. McDermott, P. S. Shaffer, and the Physics Education Group at the University of Washington, Tutorials in Introductory Physics, First Edition, Prentice Hall, Upper Saddle River, NJ, 2002.

[6] F. Biddulph and R. Osborne, Res. Sci. Ed. 14, 114-124 (1984). 